\title{
Female Gender Attenuates Cytokine and Chemokine Expression and Leukocyte Recruitment in Experimental Rodent Abdominal Aortic Aneurysms
}

\author{
INDRANIL SINHA, BRENDA S. CHO, KAREN J. ROELOFS, \\ JAMES C. STANLEY, PETER K. HENKE, \\ AND GILBERT R. UPCHURCH, JR. \\ Department of Surgery, Jobst Vascular Research Laboratories, Section of \\ Vascular Surgery, University of Michigan, Ann Arbor, MI 48109-0329
}

\begin{abstract}
Female gender appears to be protective in the development of abdominal aortic aneurysms (AAAs). This study sought to identify gender differences in cytokine and chemokine expression in an experimental rodent AAA model. Male and female rodent aortas were perfused with either saline (control) or elastase to induce AAA formation. Aortic diameter was determined and aortic tissue was harvested on postperfusion days 4 and 7. Cytokine and chemokine gene expression was examined using focused gene arrays. Immunohistochemistry was used to quantify aortic leukocyte infiltration. Data were analyzed by Student's $t$-tests and ANOVA. Elastase-perfused female rodents developed significantly smaller aneurysms compared to males by day $7(93 \pm 10 \%$ vs. $201 \pm 25 \%$, $P=0.003)$. Elastase-perfused female aortas exhibited a fivefold decrease in expression of the BMP family and ligands of the TNF superfamily compared to males. In addition, the expression of members of the TGF $\beta$ and VEGF families were three to fourfold lower in female elastase-perfused aortas compared to males. Multiple members of the interleukin, CC chemokine receptor, and $\mathrm{CC}$ ligand families were detectable in only the male elastase-perfused aortas. Female elastase-perfused aortas demonstrated a corollary twofold lower neutrophil count (females: $17.5 \pm 1.1$ PMN/HPF; males: $41 \pm 5.2$ neutrophils/HPF, $P=0.01$ ) and a 1.5 -fold lower macrophage count (females: $12 \pm 1.1$ macrophages/HPF; males: $17.5 \pm 1.1$ macrophages/HPF, $P=0.003$ ) compared to male elastaseperfused aortas. This study documents decreased expression of multiple cytokines and chemokines and diminished leukocyte trafficking in female rat aortas compared to male aortas following elastase perfusion.
\end{abstract}

Address for correspondence: Gilbert R. Upchurch, Jr., M.D., University of Michigan Hospital, 2210 THCC, 1500 East Medical Center Drive, Ann Arbor, MI 48109-0329. Voice: 734-936-5790; fax: 734647-9867.

e-mail: riversu@umich.edu

Ann. N.Y. Acad. Sci. 1085: 367-379 (2006). (C) 2006 New York Academy of Sciences.

doi: 10.1196/annals.1383.027 
These genes may contribute to the gender disparity seen in AAA formation.

KEYwORDS: AAAs; gender; cytokine; chemokine; gene array

\section{INTRODUCTION}

Male gender is a well-established risk factor in the formation of abdominal aortic aneurysms (AAA). Clinically, men form AAAs at a 4:1 ratio compared to women. ${ }^{1}$ However, AAA development becomes accelerated in women postmenopausal. ${ }^{2}$ These observations support the tenet that circulating estrogens may play a protective role in limiting pathologic inflammation in the aortic wall associated with AAA formation. ${ }^{3}$ In this regard, it is known that estrogen attenuates the inflammatory response and decreases leukocyte infiltration in many nonaneurysmal cardiovascular diseases. ${ }^{4}$

The mechanisms underlying the gender disparity in AAA formation are not well understood. Few studies have examined the role of gender in experimental AAA models. We reported the incidence and size of AAAs are significantly less in female rodents compared to males. ${ }^{5}$ In this regard, female aortas also exhibit significantly decreased aortic wall macrophage secreting matrix metalloproteinase (MMP)-9, an enzyme critical for AAA formation. ${ }^{5,6}$ In a separate study, tamoxifen, a selective estrogen receptor modulator, also attenuated experimental AAA formation and limited neutrophil infiltration. ${ }^{7}$ The present study was undertaken to determine if gender disparities in cytokine and chemokine expression contribute to decreased leukocyte trafficking in experimental female AAAs.

\section{MATERIALS AND METHODS}

All rats were obtained from Charles River Laboratories (Wilmington, MA, USA). All experiments were approved by the University of Michigan Universal Committee on the Use and Care of Animals (UCUCA No. 8566).

\section{Aneurysm Induction}

Male and female Sprague-Dawley rats, weighing 190-210 g, underwent elastase perfusion as previously described. ${ }^{8}$ Briefly, rats were anesthetized with $1-2 \%$ isoflurane inhalation and the infrarenal aorta was isolated. Temporary control of the aorta was obtained, lumbar branches were ligated, and an aortotomy was made near the bifurcation using a 30 -gauge needle. The aorta was then perfused for $30 \mathrm{~min}$ with either $2 \mathrm{~mL}$ of normal saline or 12 units of 
porcine pancreatic elastase (Sigma, St. Louis, MO, USA) in $2 \mathrm{ml}$ of normal saline.

Infrarenal aortic diameters were measured prior to perfusion, immediately postperfusion, and prior to harvest. This was accomplished using a Spot Insight Color Optical Camera (Diagnostic Instruments, Inc., Sterling Heights, MI, USA) attached to an operating microscope (Nikon, Melville, NY, USA) utilizing Image Pro Express software (Media Cybernetics, Inc., Silver Springs, MD, USA). Aortas harvested on 4 and 7 days postperfusion ( $n=5$ to 6 at each time point for both saline controls and elastase-perfused animals) were subjected to RNA (seems like total RNA was used) extraction and paraformaldehyde fixation.

\section{Gene Arrays}

Total RNA was isolated by treatment of aortic segments harvested on postperfusion day 4 with TRIzol reagent (Life Technologies, Rockville, MD, USA). cDNA was produced by reverse transcription using oligo-(dT) primer and M-MLV reverse transcriptase (Life Technologies). Two micrograms of total RNA from each animal ( $n=4$ per gender, per array, for both saline controls and elastase-perfused aortas) were obtained for microarray experiments. RNA labeling was accomplished using the SuperArray Amplolabeling Linear Polymerase Reaction kit (SuperArray Bioscience Corp., Frederick, MD, USA) according to the manufacturer's specifications. Hybridization was performed using Oligo-GE Q-series Rat Gene Arrays, ORN-021 (Rat Cytokine) and ORN-022 (Rat Chemokines and Receptors) per provided protocol. Gene expression was standardized to all housekeeping genes included in the corresponding array. Gene arrays were visualized using the provided Superarray Chemiluminescent Detection Kit. Photographs were obtained using a CCD camera. All data analysis was done using online GEArray Expression Analysis Suite software (http://geasuite.superarray.com).

\section{Immunohistochemistry}

Aortic tissue was fixed in 4\% paraformaldehyde for $20 \mathrm{~h}$, transferred to $70 \%$ ethanol, and subsequently embedded in paraffin for immunohistochemistry. Aortic tissue deparaffinization and rehydration were performed using xylene and graded alcohols. The sections were stained for macrophages and neutrophils using the following procedures. The aortic sections were deparaffinized in xylene and rehydrated in graded alcohols.

For neutrophil staining, blocking buffer was then applied to prevent nonspecific binding. Rabbit anti-rat neutrophil primary antibody (Accurate Chemical and Scientific Corporation, Westbury, NY, USA) was used for staining, 
followed by an antirabbit IgG biotinylated secondary antibody and avidinbiotin complex-AP reagent available in the Rabbit IgG Alkaline Phosphatase ABC Kit (Vector Laboratories, Burlingame, CA, USA). The sections were then visualized using the Vector Red Alkaline Phosphatase Substrate Kit I (Vector Laboratories) and counterstained with Hematoxylin QS (Vector Laboratories).

Macrophage staining entailed heat-induced epitope retrieval using $10 \mathrm{mM}$ sodium citrate, $\mathrm{pH}$ 6.0, in a microwave. The sections were subsequently incubated with $3 \%$ hydrogen peroxide in methanol to block endogenous peroxidase activity, followed by a blocking buffer to prevent nonspecific binding. Mouse antirat CD68 primary antibody (Serotec, Raleigh, NC, USA) was used for staining, followed by an antimouse IgG biotinylated secondary antibody and an avidin-biotin-HRP complex, available in the Mouse IgG Elite Vectastain ABC Kit (Vector Laboratories). Sections were then visualized using a DAB Peroxidase Substrate Kit (Vector Laboratories) and counterstained with Hematoxylin QS (Vector Laboratories). All cell counts were done by a trained, blinded observer in 10 separate $100 \times$ high-powered fields (HPF) of both the adventitia and media. A mean value was then calculated for positively stained cells in each animal.

\section{Statistics}

Data analysis was performed using nonpaired Student's $t$-tests and analysis of variance (ANOVA) with significance set as $P<0.05$. All data are expressed as the mean \pm standard error of the mean (SEM). Statistical analysis was performed using SigmaStat (Version 2.03, Copyright 1992-1997, SPSS Inc.).

\section{RESULTS}

\section{Female Aortas Form Smaller Experimental AAAs}

Saline-perfused aortas did not form aneurysms in either gender. Mean diameters of elastase-perfused male aortas increased $88 \pm 2.7 \%$ and $201 \pm 25.1 \%$ by postperfusion days 4 and 7 , respectively. In contrast, the mean diameters of female elastase-perfused aortas increased $65 \pm 4.4 \%(P=0.002)$ and $93 \pm$ $10 \%(P=0.003)$ by postperfusion days 4 and 7 , respectively (FIG. 1$)$. Six of six elastase-perfused male aortas formed aneurysms as defined by a $100 \%$ increase in aortic diameter by postperfusion day 7 in contrast to one of six elastase-perfused female aortas. 


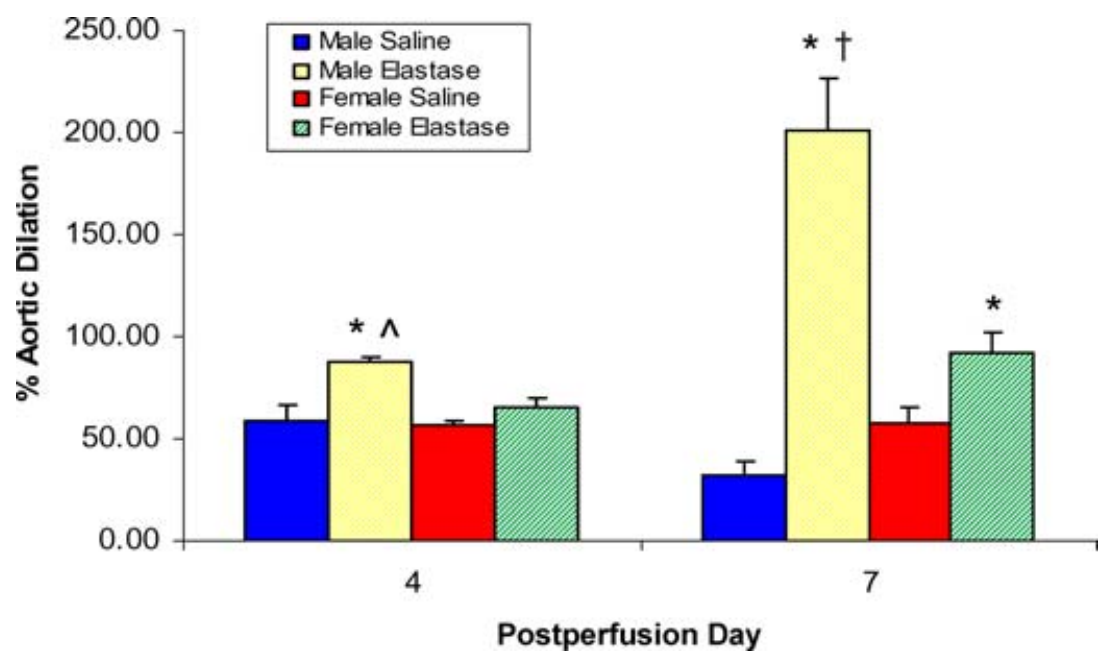

FIGURE 1. Attenuated experimental AAA formation in females. Elastase-perfused male aortas were significantly larger than elastase-perfused female aortas $(\mathrm{N}=5$ to 6 per group at each day of harvest) by postoperative day 7 . Six of six elastase-perfused male aortas formed aneurysms by day 7 as defined by a $100 \%$ increase in aortic diameter. In contrast, only one of six female elastase-perfused aorta became aneurysmal by postperfusion day 7. No saline-perfused aortas became aneurysmal in either gender. ${ }^{*} P<0.05$ for elastaseperfused aortas compared to same postperfusion day saline perfused control for that gender, ${ }^{\dagger} P<0.05$ for elastase-perfused male aortas compared to same postperfusion day elastaseperfused female aortas.

\section{Female Aortas Exhibited Decreased Expression of Multiple Cytokines and Chemokines by Gene Array on Postperfusion Day 4}

No appreciable cytokine or chemokine expression was demonstrated in saline-perfused male or female control aortas on postperfusion day 4. Elastaseperfused female aortas compared to elastase-perfused male aortas exhibited a fivefold decrease in expression of multiple genes in the bone morphogenetic protein (BMP) family, including BMP1, BMP6, BMP7, and BMP15, and tumor necrosis factor superfamily ligands (TNFSF), specifically TNFSF4, TNFSF6, TNFSF9, and TNFSF14. The transforming growth factor beta (TGF $\beta$ ) family, specifically TGF $\beta 1$, TGF $\beta 2$, and TGF $\beta 3$, as well as vascular endothelial growth factor VEGF1 and VEGF2, were three to fourfold lower in female elastase-perfused aortas compared to male elastase-perfused aortas. Multiple members of the interleukin (IL) family were detectable in only the male elastase-perfused aortas including IL8 receptor $\alpha$ and $\beta$, IL11, IL12, and IL13. Similarly, multiple genes of the CC chemokine receptor (CCR) family, specifically CCR2, CCR6, CCR7, and CCR9 as well as the CC ligand 
TABLE 1. Female gender and decreased cytokine and chemokine expression in experimental AAA formation. Note decreased cytokine and chemokine production in female elastase-perfused aortas on postperfusion day 4. ND (none detectable) denotes expression was present in males, but not detectable in female aortas

\begin{tabular}{|c|c|}
\hline Cytokine/Chemokine & Fold decrease in females \\
\hline \multicolumn{2}{|c|}{ Bone morphogenetic protein (BMP) family } \\
\hline BMP1 & 6.1 \\
\hline BMP6 & 6.3 \\
\hline BMP7 & 5.6 \\
\hline BMP15 & 6.3 \\
\hline \multicolumn{2}{|c|}{ C-C Chemokine ligand family } \\
\hline CCL24 & ND \\
\hline CCL25 & ND \\
\hline CCL28 & ND \\
\hline \multicolumn{2}{|c|}{ C-C Chemokine receptor family } \\
\hline CCR2 & ND \\
\hline CCR7 & ND \\
\hline CCR8 & ND \\
\hline \multicolumn{2}{|l|}{ Interleukin (IL) family } \\
\hline IL1 & 6.2 \\
\hline IL2 & 6.1 \\
\hline IL3 & 4.9 \\
\hline IL5 & 4.9 \\
\hline IL7 & 5.9 \\
\hline IL8 $\alpha$ & ND \\
\hline IL8 $\beta$ & ND \\
\hline IL11 & 6 \\
\hline IL12 & 6.3 \\
\hline \multicolumn{2}{|c|}{ Transforming growth factor (TGF) family } \\
\hline TGF $\beta 1$ & 4.1 \\
\hline TGF $\beta 2$ & 4.2 \\
\hline \multicolumn{2}{|c|}{ Tumor necrosis factor (TNF) family } \\
\hline TNFSF4 & ND \\
\hline TNFSF6 & ND \\
\hline TNFSF9 & ND \\
\hline TNFSF15 & ND \\
\hline \multicolumn{2}{|c|}{ Vascular endothelial growth factor family } \\
\hline VEGF1 & 3.6 \\
\hline VEGF2 & 3.6 \\
\hline
\end{tabular}

(CCL) family, specifically CCL3, CCL5, CCL24, CCL25, and CCL28, were detectable in male elastase-perfused aortas, but not in female elastase-perfused aortas (TABLE 1).

Female Aortas Exhibit Decreased Neutrophil and Macrophage Infiltration

Minimal neutrophil and macrophage infiltration was detected in both male and female saline perfumed aortas. Male elastase-perfused aortas demonstrated $13.7 \pm 5.9$ positively staining cells/HPF in males compared to $5.0 \pm 0.5$ 
neutrophils/HPF $(P=0.09)$ in females on postperfusion day 4. By postperfusion day 7 , male elastase-perfused aortas demonstrated a twofold greater neutrophil count (40.75 $\pm 5.2 \mathrm{PMN} / \mathrm{HPF})$ in comparison to female elastaseperfused aortas (17.5 $\pm 1.1 \mathrm{PMN} / \mathrm{HPF}, P=0.01)$ (FIgs. 2A and B). Male elastase-perfused aortas also demonstrated $12.2 \pm 5.1$ and $17.5 \pm 1.1$ CD68 positive cells (macrophages)/HPF on postperfusion days 4 and 7, respectively. Number of macrophages was significantly fewer in females $(4.7 \pm$ 1.1 macrophages/HPF on postperfusion day $4, P=0.2$, and $11.8 \pm 1.1$ macrophages/HPF on postperfusion day $7, P=0.003$ ) (Figs. 3A and B).

\section{DISCUSSION}

This study confirms that female rodents develop significantly smaller experimental AAAs compared to males. Cytokine and chemokine gene arrays identified several cytokine and chemokine families, which were differentially expressed by gender including BMP, TNF, TGF $\beta$, IL, CCR, and CCL gene families. Furthermore, female rat aortas exhibited significantly fewer infiltrating neutrophils and macrophages by postperfusion day 7 .

It is possible that the known effect of estrogen in females on inflammatory processes in cardiovascular diseases may be associated with the observed decreased leukocyte cell infiltration in females and their apparent resistance to AAA formation. ${ }^{9}$ Multiple studies have shown estrogen plays a protective role by attenuating inflammatory cell mediated damage by downregulating cytokine and chemokine expression. In two separate AAA models, female gender has been shown to limit experimental AAA formation, at least in part by limiting neutrophil and macrophage infiltrate. ${ }^{4}{ }^{10}$ In addition, Miller et al. observed that estrogen attenuation of the early inflammatory response following endoluminal vascular injury was associated with decreased expression of several cytokines and chemokines, especially the CXC chemokine family. ${ }^{11}$ This was accompanied with decreased neutrophil and macrophage infiltration and vasoprotection through an apparent anti-inflammatory pathway. ${ }^{11}$ Estrogen is also known to decrease myocardial inflammation and damage following ischemia-reperfusion injury by downregulating proinflammatory cytokines such as TNF $\alpha$, IL1 $\beta$, and IL6. ${ }^{12}$

Estrogen also attenuates inflammation-mediated damage in nonvascular tissue. Santizo et al. demonstrated that chronic estrogen depletion enhanced leukocyte adhesion in the rat cerebral circulation and suggested this was a mechanism underlying increased neural damage in ovariectomized females as compared to intact females. ${ }^{13}$ In addition, estrogen has been observed to enhance wound healing through an anti-inflammatory effect by limiting neutrophil and macrophage infiltration and suppressing the production of numerous cytokines including $\mathrm{TNF} \alpha$ and macrophage migration inhibitory factor (MIF). ${ }^{14}$ 
Estrogen is likely to decrease cytokine and chemokine expression through multiple mechanisms. First, estrogen decreases p38 mitogen activated protein kinase (MAPK), a crucial upstream proinflammatory regulator of numerous cytokines, including TNF $\alpha$, IL1, IL4, IL6, and IL8. ${ }^{15}$ Wang et al. demonstrated decreased p38 MAPK phosphorylation (activation) in control females compared to oophorectomized females and control males following ischemia/reperfusion injury. ${ }^{12}$ Second, estrogen also decreases the activity of nuclear factor $\kappa \mathrm{B}(\mathrm{NF \kappa B})$, a key proinflammatory transcription factor. ${ }^{16} \mathrm{Fi}$ nally, estrogen decreases oxidant stress, a stimuli for the production of multiple inflammatory cytokines in ischemia/reperfusion injury. ${ }^{15}$

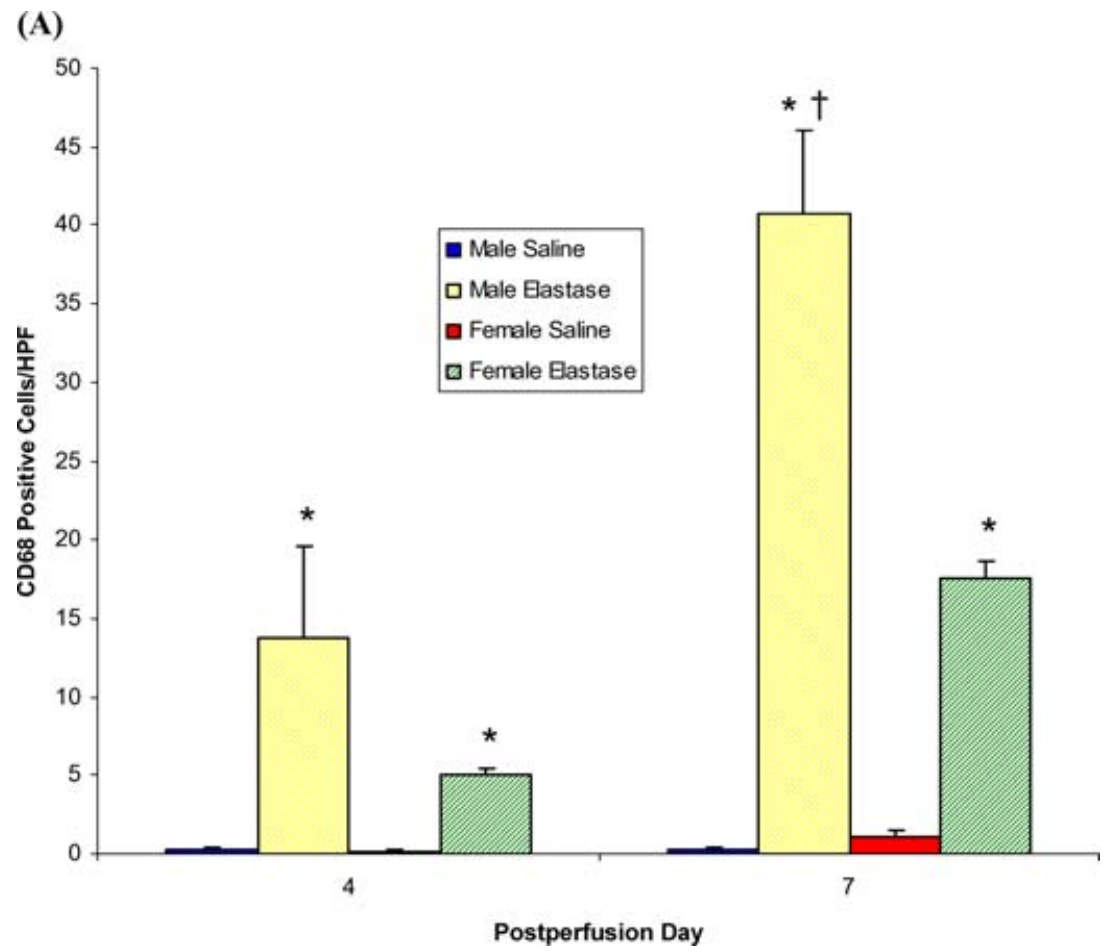

FIGURE 2. Female gender and decreased aortic wall neutrophil infiltration. (A) Immunohistochemistry $(100 \times)$ by postoperative day 7 revealed significantly increased neutrophil infiltration in the adventitia of male aortas compared to female aortas. Few positively stained cells were identified in saline-perfused controls of either gender. ${ }^{*} P<0.05$ for elastase-perfused aortas compared to same postperfusion day saline-perfused control for that gender, ${ }^{\dagger} P<0.05$ for elastase-perfused male aortas compared to same postperfusion day elastase-perfused females, $\mathrm{N}=4$ per group at each day of harvest. (B,C) Representative images of positively staining cells (arrows) in the adventitia of elastase-perfused male (B) and fewer positively staining cells in the female aortas $(\mathbf{C})(100 \times)$. [Saline-perfused aortas not shown.] 
(B)

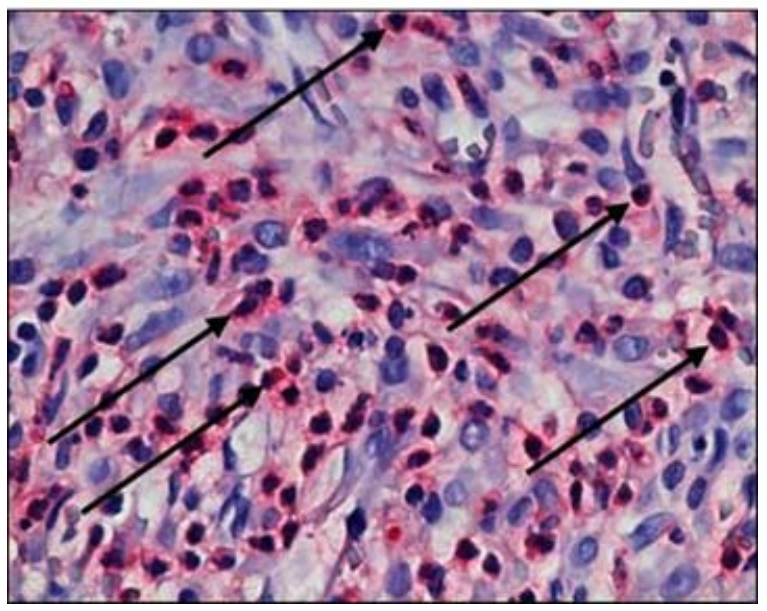

(C)

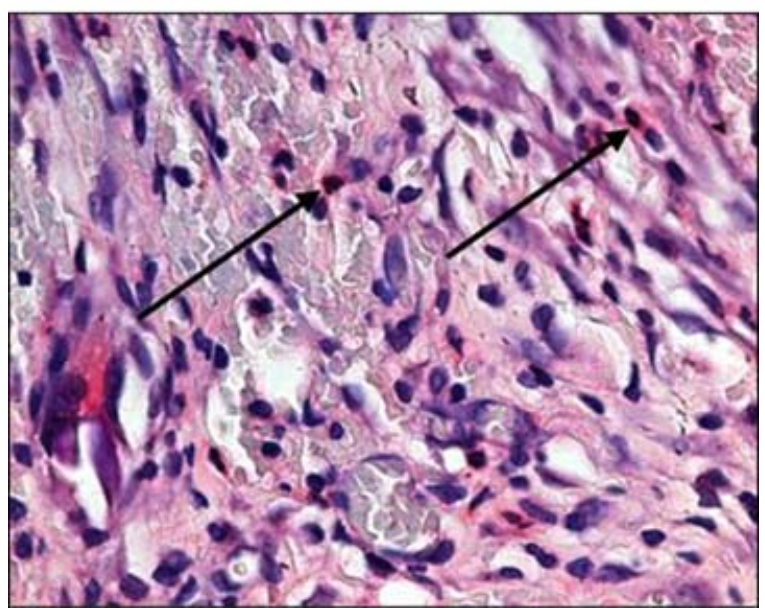

FIGURE 2. Continued.

The present intervention demonstrates multiple cytokine families that were differentially regulated by gender, although their role in AAA pathogenesis has not been thoroughly investigated. Others have provided some insight into this subject. Dai et al. have previously shown that adenoviral delivery mediated exogenous overexpression of TGF- $\beta 1$ stabilized preformed experimental AAAs. ${ }^{17}$ However, no studies to date have examined gender differences in TGF $\beta$ regulation in experimental AAAs. Similarly, the TNF superfamily is known to have a role in AAA pathogenesis, but no studies have assessed 
gender difference in AAA formation. ${ }^{6}$ Kobayashi et al. demonstrated VEGF to be upregulated in AAAs, but a role in AAA pathogenesis remains unclear. ${ }^{18}$ Certain ILs are known to be critical to AAA formation, but no evidence exists for the roles of IL11, IL12, or IL13. ${ }^{6}$

A limited number of investigators have examined the role of chemokines in AAA formation. Zhao et al. demonstrated that macrophage inhibitory protein (MIP) $1 \alpha$ (also known as CCL3), which was differentially expressed by gender in the present study, and MIP2 (CXCL2) were important in experimental AAA formation. ${ }^{19}$ Similarly, Yamagishi et al. observed an elevenfold increase in CXCR2 production in human AAAs compared to control healthy aortas. ${ }^{20}$ However, no studies to date have elucidated gender differences in the patterns of chemokine expression.

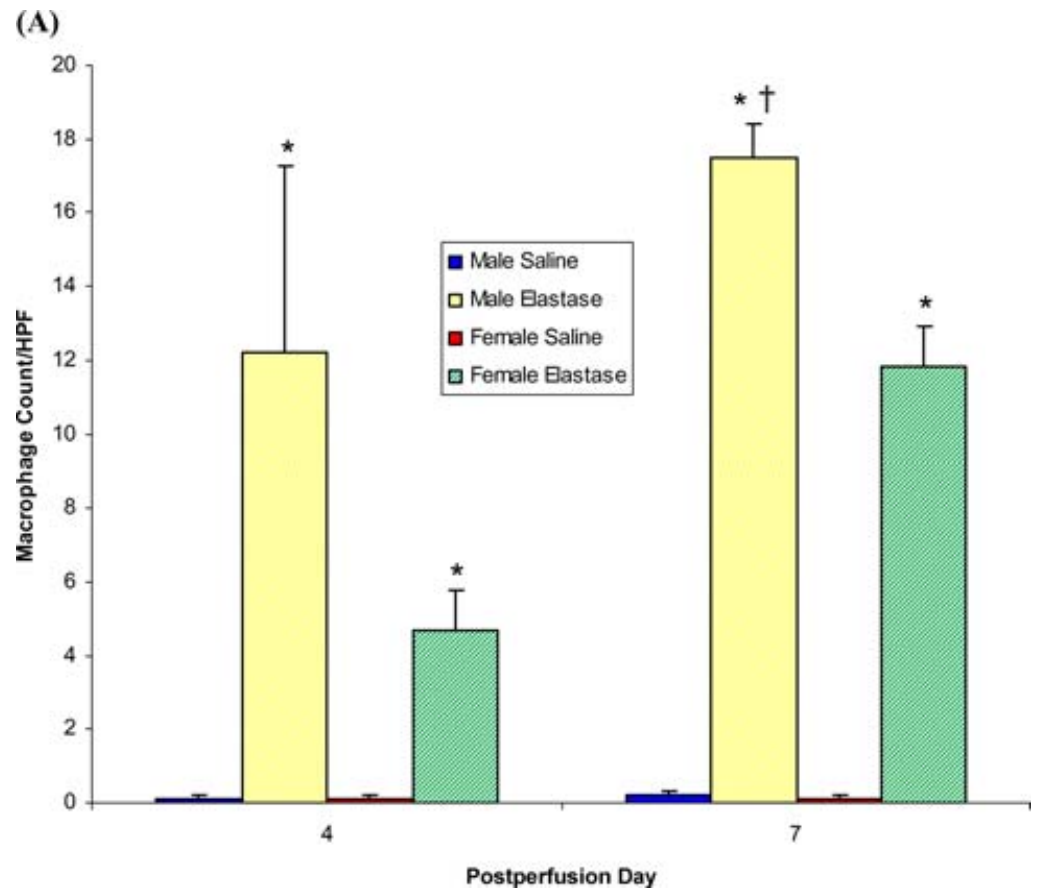

FIGURE 3. Female gender and decreased aortic wall macrophage infiltration. (A) Immunohistochemistry $(100 \times)$ by postoperative day 7 revealed significantly increased macrophage infiltration in the adventitia of male aortas compared to female aortas. Minimally positively stained cells were identified in saline-perfused controls of either gender. ${ }^{*} P<0.05$ for elastase-perfused aortas compared to same postperfusion day saline-perfused control for that gender, ${ }^{\dagger} P<0.05$ for elastase-perfused male aortas compared to same postperfusion day elastase-perfused females, $\mathrm{N}=4$ per group at each day of harvest. (B,C) Representative images of positively CD68 staining cells (arrows) in the adventitia of elastase-perfused male (B) and minimal positively staining cells in the female aortas (C) $(100 \times)$. (Saline-perfused aortas not shown.) 
(B)

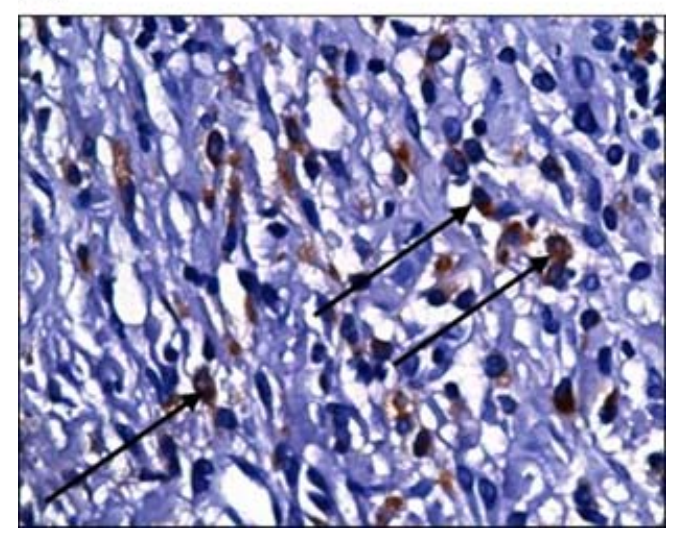

(C)

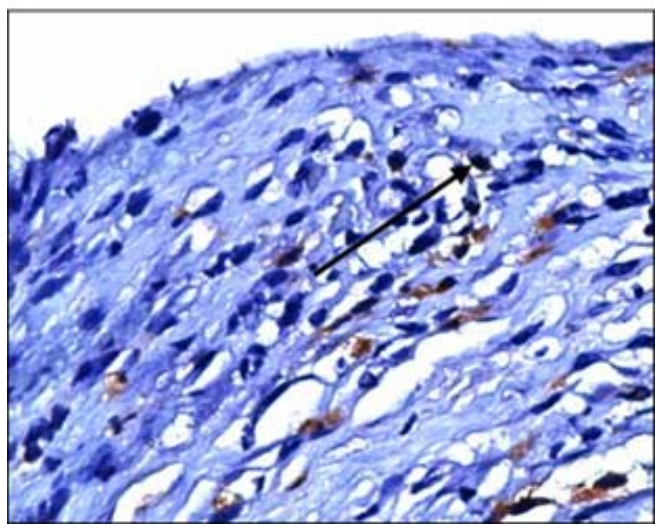

FIGURE 3. Continued.

The present investigation supports the speculation that a number of cytokines and chemokines may be differentially regulated by gender and may contribute to vessel wall injury. More reliable polymerase chain reaction or northern blotting data will be required to confirm these observations. This study also only examined postperfusion day 4 and therefore does not take into account genes that may be upregulated either earlier or later in experimental AAA formation. Additionally, it will be necessary to determine if gender differences in cytokine and chemokine differences are intrinsic to the wall or secondary to diminished leukocyte trafficking into female aortas. Nevertheless, this study has identified multiple gene families that deserve further consideration for a potential role in gender differences during AAA formation. Furthermore, continuing research is needed to more fully elucidate the mechanism by which estrogen acts to decrease cytokine and chemokine expression. A better understanding 
of gender disparities may ultimately lead to new pharmacological targets for the treatment of AAAs.

\section{ACKNOWLEDGMENTS}

This work was supported by NIH KO8 (HL67885-02) (GRU), Von Liebig Award-Lifeline Foundation (GRU), the Lifeline Medical Student Research Award (IS), the Griswold and Margery H. Ruth Alpha Omega Alpha Medical Student Research Fellowship (IS), and the Jobst Foundation.

\section{REFERENCES}

1. Singh, K., K.H. BonaA, B.K. Jocobsen, et al. 2001. Prevalence and risk factors for abdominal aortic aneurysms in a population based study: the Tromso study. Am. J. Epidemiol. 154: 236-244.

2. Bengtsson, H., B. Sonesson \& D. Bergvist. 1996. Incidence and prevalence of AAA. N. Y. Acad. Sci. 800: 1-24.

3. LaVecchia, C., A. DeCarli, S. Franceschi, et al. 1987. Menstrual and reproductive factors and the risk of myocardial infarction in women under fifty-years of age. Am. J. Obstet. Gynecol. 157: 1108-1112.

4. BAKER, L., K.K. MELDRUM, M. WANG, et al. 2003. The role of estrogen in cardiovascular disease. J. Surg. Res. 115: 325-344.

5. Ailawadi, G., J.L. Eliason, K.J. Roelofs, et al. 2004. Gender differences in experimental aneurysm formation. Arterioscler Thromb. Vasc. Biol. 24: 21162122.

6. Ailawadi, G., J.L. Eliason \& G.R. UpchuRch. 2003. Current concepts in the pathogenesis of abdominal aortic aneurysms. J. Vasc. Surg. 38: 584-588.

7. Grigoryants, V., K.K. Hannawa, C.G. Pearce, et al. 2005. Tamoxifen upregulates catalase production, inhibits vessel wall neutrophil recruitment, and attenuates development of experimental abdominal aortic aneurysms. J. Vasc. Surg. 41: 108-114.

8. AnidjaR, S., J.L. Salzmann, D. Gentric, et al. 1990. Elastase induced experimental aneurysms in rats. Circulation 82: 973-981.

9. LeINWAND, L.A. 2003. Sex is a potent modifier of the cardiovascular system. J. Clin. Invest. 112: 302-307.

10. Martin-McNulty, B., D.M. Tham, C. DA, et al. 2003. $17 \beta$-estradiol attenuates development of angiotensin II-induced aortic abdominal aneurysm in apolipoprotein E-deficient mice. Arterioscler Thromb. Vasc. Biol. 23: 16271632.

11. MilLER, A.P., W. FenG, D. XING, et al. 2004. Estrogen modulated inflammatory mediator expression and neutrophil chemotaxis in injured arteries. Circulation 110: $1664-1669$.

12. WANG, M., B.M. TsaI, K.M. ReIGER, et al. 2006. 17- $\beta$-estradiol decreases p38 MAPK-mediated myocardial inflammation and dysfunction following acute ischemia. J. Mol. Cell. Cardiol. 40: 205-212.

13. Santizo, R. \& D.A. Pelligrino. 1999. Estrogen reduces leukocyte adhesion in the cerebral circulation of female rats. J. Cereb Blood Flow Metab. 19: 1061-1065. 
14. Ashcroft, G.S., S.J. Mills, K. Lei, et al. 2003. Estrogen modulated cutaneous wound healing by downregulating macrophage migration inhibitory factor. J. Clin. Invest. 111: 1309-1318.

15. Kher, A., M. WAng, B.M. Tsai, et al. 2005. Sex differences in the myocardial inflammatory response to acute injury. Shock 23: 1-10.

16. Deshrande, R., H. Khalili, R.G. Pergolizzi, et al. 1997. Estradiol down-regulates LPS-induced cytokine production and NFKB activation in murine macrophages. Am. J. Reprod. Immunol. 38: 46-54.

17. Dai, J., F. Losy, A.M. Guinault, et al. 2005. Overexpression of transforming growth factor-beta1 stabilizes already formed aortic aneurysms: a first approach to induction of functional healing by endovascular gene therapy. Circulation 112: 1008-1015.

18. Kobayashi, M., J. Matsubara, M. Matshushita, et al. 2002. Expression of angiogenesis and angiogenic factors in human aortic vascular disease. J. Surg. Res. 106: 239-245.

19. ZhaO, L., M.P. Moos, R. Grabner, et al. 2004. The 5-lipoxygenase pathway promotes pathogenesis of hyperlipidemia-dependent aortic aneurysm. Nat. Med. 10: 966-973.

20. Yamagishi, M., T. Higashikata, H. Ishibashi-Ueda, et al. 2005. Sustained upregulation of inflammatory chemokine and its receptor in aneurysmal and occlusive atherosclerotic disease: results from tissue analysis with cDNA macroarray and real-time reverse transcriptional polymerase chain reaction methods. Circ. J. 69: 1490-1495. 